\title{
Oscillating magnetoresistance in diluted magnetic semiconductor barrier structures
}

\author{
Kai Chang* and J. B. Xia \\ NLSM, Institute of Semiconductors, Chinese Academy of Sciences, Beijing 100083, China \\ F. M. Peeters ${ }^{\dagger}$ \\ Department of Physics, University of Antwerp (UIA), Universiteitsplein 1, B-2610 Antwerpen, Belgium
}

(Received 9 July 2001; published 8 March 2002)

\begin{abstract}
Ballistic spin polarized transport through diluted magnetic semiconductor single and double barrier structures is investigated theoretically using a two-component model. The tunneling magnetoresistance (TMR) of the system exhibits oscillating behavior when the magnetic field is varied. An interesting beat pattern in the TMR and spin polarization is found for different nonmagnetic semiconductor/diluted magnetic semiconductor double barrier structures which arises from an interplay between the spin-up and spin-down electron channels which are split by the $s$ - $d$ exchange interaction.
\end{abstract}

DOI: 10.1103/PhysRevB.65.115209

PACS number(s): 75.50.Pp, 72.10.- d, 72.90.+y, 84.32.-y

Spin polarized transport in solid-state systems has generated intense interest as it is the crucial ingredient for spintronics $^{1,2}$ and several quantum computation schemes. ${ }^{3,4}$ For these applications, a basic requirement is to produce, and sustain, high spin-polarized currents in semiconductors for sufficient long times. Several schemes have been proposed to produce spin injection in semiconductors, such as electrons injected from a ferromagnetic metal into a semiconductor, ${ }^{5-8}$ but the change in device resistance for parallel and antiparallel magnetization is very small. Diluted magnetic semiconductors (DMS) provides us with a new system in which spin dependent optical and transport properties are expected. The spin-dependent transport and optical properties in DMS systems arise from the $s$ - $d$ exchange interaction between the conduction electron and the localized $3 d^{5}$ electrons of the Mn ions which lifts the degeneracy of the spin-up and spindown electron and hole states. ${ }^{9}$

Spin dependent optical properties in a DMS spin superlattice was proposed ${ }^{10}$ and realized. ${ }^{11}$ Spin-dependent tunneling through a DMS junction was also studied theoretically within a mean-field approximation. ${ }^{12-15}$ Very recently, experiments have demonstrated that a robust spin injection through a diluted magnetic semiconductor junction $\mathrm{Be}_{x} \mathrm{Mn}_{y} \mathrm{Zn}_{1-x-y} \mathrm{Se},{ }^{16}$ and a Mn doped $p$-type GaAs spin aligner was possible in which holes were injected into GaAs in the presence of an in-plane magnetic field. ${ }^{17}$ Spin polarization of the injected carriers was detected by the emitted circular polarized light from the holes which recombined with the electrons in the nonmagnetic semiconductor (NMS) quantum wells. Spin coherence can be maintained in semiconductors over large distances $(\geqslant 100 \mu \mathrm{m})$ and for long time (up to nanoseconds). ${ }^{18}$

In this work, we report an interesting oscillating tunneling magnetoresistance (TMR) and spin polarization (SP) through NMS/DMS structures. The DMS structure is similar to the sample used in the magneto-optical study of Ref. 11. We find theoretically that the TMR for the double barrier structure oscillates with increasing magnetic field and exhibits a peculiar beat pattern. The underlying physics of the phenomena arises from the interplay of the spin-up and the spin-down channels which are split by the $s-d$ exchange interaction.
Note that band-structure effects and spin-orbit interaction are not very efficient spin-flip processes for electrons. ${ }^{19}$ Egues et al. ${ }^{13}$ first presented theoretical results on spin filtering and magnetoresistance through ballistic tunneling junctions. In their seminal work, a peculiar beat pattern in the double NMS barriers with DMS contacts is similar to ours, and they pointed out that the beat pattern is directly related to the peculiar overlap of the $s$ - $d$ spin-split transmission channel. Here we additionally studied double DMS barriers with NMS contacts which we found to exhibit also beat patterns. Furthermore, in our calculation we used a different approach which is more easily applicable to more complicated cases, such as, e.g., the case with an electric bias. In this work we prove that the beat pattern which arises from the superposition of the spin-up and the spin-down channel is a rather general phenomena and can be observed in either NMS double barrier structures with DMS contacts or DMS barriers with NMS contacts. Instead of $\mathrm{ZnSe}_{\mathrm{Zn}} \mathrm{n}_{1-x} \mathrm{Mn}_{x} \mathrm{Se}$ structures used in the calculation of Ref. 13, we propose $\mathrm{Cd}_{1-y} \mathrm{Mg}_{y} \mathrm{Te} / \mathrm{Cd}_{1-x} \mathrm{Mn}_{x} \mathrm{Te}$ structures to realize single and double $\mathrm{NMS}\left(\mathrm{Cd}_{1-y} \mathrm{Mg}_{y} \mathrm{Te}\right)$ barrier structures with DMS contacts $\left(\mathrm{Cd}_{1-x} \mathrm{Mn}_{x} \mathrm{Te}\right)$.

Consider a spin unpolarized electron injected into a NMS/ DMS/NMS single or double barrier structure in the presence of a perpendicular magnetic field. Due to the $s-d$ exchange interaction, an external magnetic field gives rise to a giant Zeeman splitting of the conduction band states which results in a striking difference of the potential profiles seen by the spin-up and the spin-down electron (see the insets of the figures). ${ }^{12}$ In this simple system, the electric current has contributions from spin-up and spin-down channels. Since the sample dimensions are much smaller than the spin coherence length, which may reach up to $100 \mu \mathrm{m}$ in semiconductors, ${ }^{16,18}$ spin-flip processes can be neglected in our system.

The model Hamiltonian of such systems is of the following form:

$$
\begin{aligned}
H= & (\mathbf{p}+e \mathbf{A})^{2} / 2 m+V(z)+1 / 2 g_{s} \mu_{B} \mathbf{s} \cdot \mathbf{B} \\
& +J_{s-d} \sum_{i} \mathbf{S}(\mathbf{r}) \cdot \mathbf{S}\left(\mathbf{R}_{i}\right) \delta\left(\mathbf{r}-\mathbf{R}_{i}\right),
\end{aligned}
$$




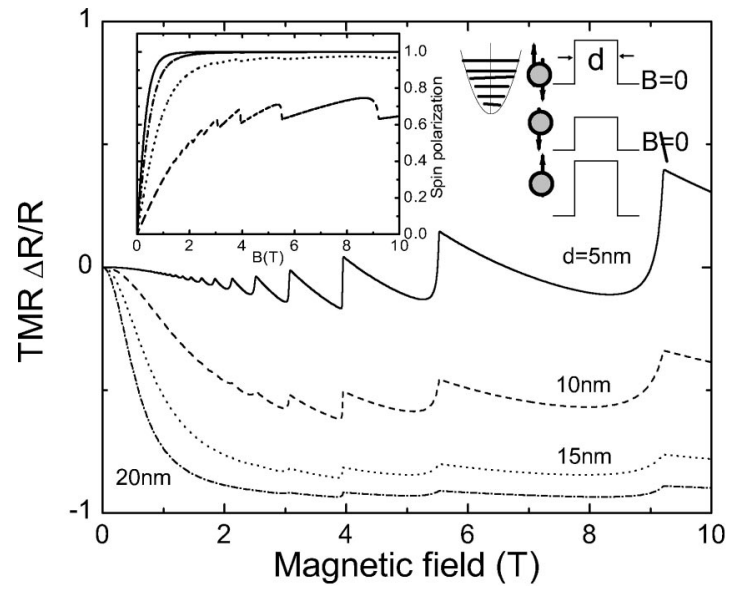

FIG. 1. The TMR $\Delta R / R$ as a function of the magnetic field for different DMS barrier thicknesses. The inset gives the spin polarization versus the magnetic field. The potential profiles for $B=0$ and $B \neq 0$ are also plotted in the figure. The solid, dashed, dotted and dash-dotted curves correspond to the different thicknesses of the DMS layer: 5, 10, 15, $20 \mathrm{~nm}$, respectively. The parameters are taken from Ref. 11 for $\mathrm{ZnSe} / \mathrm{Zn}_{1-x} \mathrm{Mn}_{x} \mathrm{Se}(x=0.07)$.

where $\mathbf{S}$ is the spin of the localized $3 d^{5}$ electrons of the $\mathrm{Mn}$ ions with $S=5 / 2$ and $\mathbf{s}$ is the electron spin. Here we assume that the magnetic ions are distributed homogeneously in the DMS layers. The exchange term with contact interaction is a strongly localized interaction and acts only on the wavefunction of the electron at a given $\mathrm{Mn}^{2+}$ site. ${ }^{20} m_{e}^{*}$ is the electron effective mass, $V(z)$ is the zero magnetic field potential profile of, e.g., $\mathrm{ZnSe} / \mathrm{Zn}_{1-x} \mathrm{Mn}_{x} \mathrm{Se}$ DMS double barrier structures. ${ }^{11}$ The vector potential is taken as $\mathbf{A}=$ $(-B y / 2, B x / 2,0)$ and therefore the magnetic field points along the $z$ axis. The third term describes the Zeeman splitting of the electron. The last term in Eq. (1) denotes the $s$-d exchange interaction between the electron and $\mathrm{Mn}$ ions. $\left\langle S_{z}\right\rangle=S B_{J}\left[S g \mu_{B} B / k_{B}\left(T+T_{0}\right)\right]$, where $B_{J}(x)$ is the Brillouin function and $S=5 / 2$ spins of the localized $3 d^{5}$ electrons of the Mn ions, $N_{0}$ is the number of cations per unit volume, $J_{s-d}=-N_{0} \alpha x_{\text {eff }}$ denotes the exchange integral

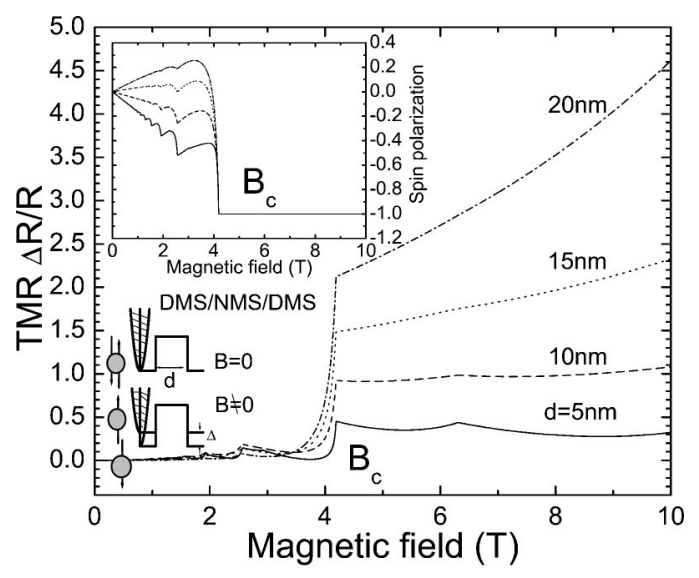

FIG. 2. The same as Fig. 1 but now for the DMS barrier with NMS contacts. The parameters are taken from Ref. 21 for $\mathrm{Cd}_{1-y} \mathrm{Mg}_{y} \mathrm{Te} / \mathrm{Cd}_{1-x} \mathrm{Mn}_{x} \mathrm{Te}(x=0.04, y=0.08)$.
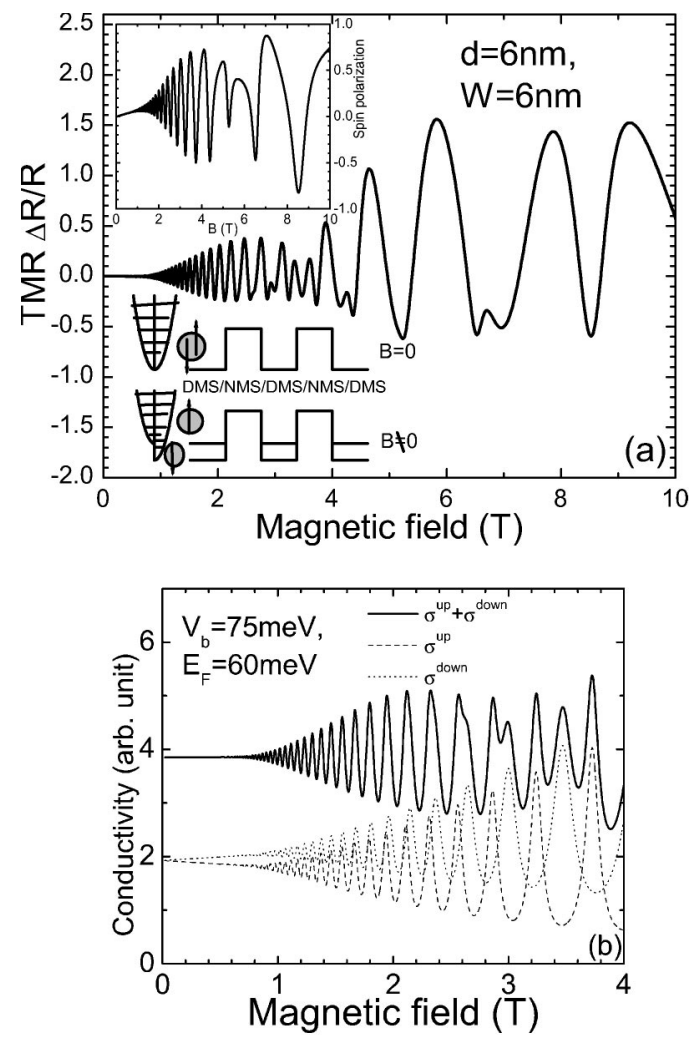

FIG. 3. (a) The TMR $\Delta R / R$ as a function of magnetic field for a NMS double barrier structure with DMS contacts. The inset shows the spin polarization for $E_{F}=60 \mathrm{meV}, V_{b}=75 \mathrm{meV}$. (b) The total conductivity (thick solid curve) and the spin-up (dashed curve) and spin-down (dotted curve) conductivities as a function of magnetic field. The parameters are taken from Ref. 21 for $\mathrm{Cd}_{1-y} \mathrm{Mg}_{y} \mathrm{Te} / \mathrm{Cd}_{1-x} \mathrm{Mn}_{x} \mathrm{Te}(x=0.04, y=0.08)$.

for the conduction band. The phenomenological parameters $x_{\text {eff }}$ (reduced effective concentration of $\mathrm{Mn}$ ) and $T_{0}$ account for the reduced single-ion contribution due to the antiferromagnetic Mn-Mn coupling, and $\mathbf{s}= \pm 1 / 2$ is the electron spin operator. The third and fourth terms in Eq. (1) can be viewed as an effective potential which is different for the spin-up and spin-down electrons (see the inset of Fig. 1). The parameters used in our calculation are taken from Ref. 11 for $\mathrm{ZnSe} / \mathrm{Zn}_{1-x} \mathrm{Mn}_{x} \mathrm{Se}$ [see Fig. $1(x=0.07)$ and Fig. $4(x=0.2)]$ and from Ref. 21 for $\mathrm{Cd}_{1-y} \mathrm{Mg}_{y} \mathrm{Te} / \mathrm{Cd}_{1-x} \mathrm{Mn}_{x} \mathrm{Te}$ [see Fig. $2(x=0.04, y=0.05)$ and Fig. $3(x=0.04, y=0.08)$ ]. The parameters used in our calculation are as follows: $\quad m_{e}^{*}=0.16 m_{0}, T_{0}=1.4 \mathrm{~K}, N_{0} \alpha=0.27 \mathrm{eV}, \quad \mathrm{V}_{b}$ $\approx 10 \mathrm{meV}$ for $\mathrm{Zn}_{0.93} \mathrm{Mn}_{0.07} \mathrm{Se}$, and $\mathrm{V}_{b} \approx 75 \mathrm{meV}$ for $\mathrm{Zn}_{0.8} \mathrm{Mn}_{0.2} \mathrm{Se} ; \quad m_{e}^{*}=0.096 m_{0}, T_{0}=3.1 \mathrm{~K}, N_{0} \alpha=0.22 \mathrm{eV}$, $\mathrm{V}_{b} \approx 10 \mathrm{meV}$ for $\mathrm{Cd}_{0.95} \mathrm{Mg}_{0.05} \mathrm{Te}_{2} / \mathrm{Cd}_{0.96} \mathrm{Mn}_{0.04} \mathrm{Te}$, and $V_{b}$ $\approx 75 \mathrm{meV}$ for $\mathrm{Cd}_{0.92} \mathrm{Mg}_{0.08} \mathrm{Te} / \mathrm{Cd}_{0.96} \mathrm{Mn}_{0.04} \mathrm{Te}$.

Electrons on the left and right hand side of the tunneling barrier can be expressed as $\psi_{L}^{\sigma}=e^{i k z} \chi_{\sigma}+r_{\sigma} e^{-i k z} \chi_{\sigma}$ and $\psi_{R}^{\sigma}$ $=t_{\sigma} e^{i k z} \chi_{\sigma}$, respectively, where the spinor $\chi_{\sigma}(\sigma=\uparrow \downarrow)$ is the spin-up $|\uparrow\rangle$ or the spin-down $|\downarrow\rangle$ state and $r_{\sigma}\left(t_{\sigma}\right)$ is the reflection (transmission) coefficient. Taking into account the boundary conditions, i.e., the continuity of the envelope function $\psi_{i}^{\sigma}$ and its derivative $\left(\psi_{i}^{\sigma}\right)^{\prime} / m_{i}$ at the interface, we 
can connect the electron wave functions $\psi_{L}^{\sigma}=T \psi_{R}^{\sigma}$ at the boundary, where $T$ is the transfer matrix which has the form $T=\Pi_{j} T_{M}^{j}=T_{M}^{n} T_{M}^{n-1} T_{M}^{n-2} \cdots T_{M}^{2} T_{M}^{1}$, with

$$
T_{M}^{j}=\left(\begin{array}{cc}
\cos k_{j} a & \frac{m_{j}}{k_{j}} \sin k_{j} a \\
-\frac{k_{j}}{m_{j}} \sin k_{j} a & \cos k_{j} a
\end{array}\right),
$$

where $k_{j}=\sqrt{2 m_{e}\left(E-V_{j}\right) / \hbar^{2}}$

When a small bias is applied across the junction, a nonequilibrium electron population will be generated. The current density $J=\Sigma_{\sigma} J^{\sigma}$ can be calculated ${ }^{22}$

$$
J^{\sigma}=\frac{e^{2} V}{2 \pi^{2} l_{B}^{2}} \sum_{n}\left(\frac{1}{2 \pi}\right) \int_{0}^{k_{n}^{F}} d k_{z}\left(-\frac{\partial f_{0}}{\partial E}\right) T^{\sigma} v_{z}^{\sigma},
$$

where $T^{\sigma}\left(n, E_{F}\right)$ is the transmission coefficient of our tunnel structure at the Fermi surface for the different spin orientations, $v_{z}^{\sigma}=\hbar k_{z}^{\sigma} / m$ is the group velocity, $1 / 2 \pi l_{B}^{2}$ is the density of state of each Landau level, $l_{B}=\sqrt{\hbar / e B}$ is the magnetic length, $f_{0}$ is the equilibrium distribution function of the conduction band electrons, and we use the approximation $-\partial f_{0} / \partial E \approx \delta\left(E-E_{F}\right)$ which is valid for $k_{B} T \ll E_{F}$. The low-temperature conductance is given by ${ }^{15}$

$$
\sigma^{\uparrow \downarrow} / \sigma_{0}=\sum_{n} T^{\uparrow \downarrow}\left(k_{n F}^{\uparrow \downarrow}\right)
$$

where $k_{n F}^{\uparrow \downarrow}=\sqrt{2 m / \hbar^{2}\left[E_{F}-\left(E_{n} \pm \Delta / 2\right)\right]}, \quad \sigma_{0}=e^{2} / 2 \pi l_{B}^{2} h, \quad \Delta$ $=J_{s-d}\left\langle S_{z}\right\rangle$ is the giant Zeeman splitting, $E_{n}=(n+1 / 2) \hbar \omega_{c}$ is the energy of the Landau level. In our formalism, the total conductivity is the sum of the conductivity of each Landau level at the Fermi surface and this for each spin state.

The degree of spin polarization (SP) of the current density is defined by

$$
P=\frac{J^{\downarrow}-J^{\uparrow}}{J^{\downarrow}+J^{\uparrow}} .
$$

Here $J^{\uparrow}\left(J^{\downarrow}\right)$ is the spin-up (spin-down) current density of the spin-polarized current.

The magnetoresistance (TMR) as a result of tunneling through the NMR/DMS structures is defined by

$$
\Delta R / R=\frac{R(B)-R(0)}{R(0)}=\frac{R(B)}{R(0)}-1=\frac{\sigma(0)}{\sigma(B)}-1 .
$$

Here $J^{\uparrow}\left(J^{\downarrow}\right)$ is the spin-up (spin-down) current density of the spin-polarized current.

Figure 1 depicts how the TMR $\Delta R / R$ varies with magnetic field in a DMS single barrier structure with NMS contacts for different thicknesses of the DMS layer. There is a striking similarity between our results and Fig. 3 of Ref. 13 . The inset shows the spin polarization $P(\mathrm{SP})$ versus magnetic fields. From this figure, we find that the TMR, on the average, decreases and oscillates with increasing magnetic field. The oscillations of the TMR and the SP are weakened by increasing the DMS barrier thickness. These oscillations are mainly attributed to the oscillation of the spin-down conduc- tivity component $\sigma^{\downarrow} / \sigma_{0}$ which is enhanced (weakened) by increasing magnetic field (the thickness of the DMS layer). The conductivity $\sigma^{\downarrow} / \sigma_{0}$ of the spin-down component is larger than that of the spin-up component, since the barrier height seen by the spin-up electron is higher than that seen by the spin-down electron due to the magnetic field-induced $s-d$ exchange interaction. Therefore the spin-polarization increases and saturates with increasing magnetic field and barrier thickness.

In Fig. 2 we plot the TMR $\Delta R / R$ as a function of magnetic field in a NMS single barrier structure with DMS contacts. There is a large difference between Figs. 1 and 2. Notice the almost steplike character in the TMR and the SP when the magnetic field approach as the critical field $B_{c}$ $\sim 4 \mathrm{~T}$ which is determined by the energy separation between the spin-up electron energy and the Fermi energy. This separation is determined by the magnetic field and the temperature [see Eq. (1)] and is independent of the thickness of the barrier. This steplike behavior arises from the competition of the spin-up and the spin-down electron conductivity. The oscillating conductivity of the spin-up (spin-down) electron decreases (increases) with increasing magnetic field, the spindown channel makes a dominant contribution to the conductivity and the spin-up channel is blocked when the magnetic field is larger than the critical field $B_{c}$ and consequently leads to a step. In the inset of Fig. 2, the spin polarization also exhibits a steplike behavior versus the magnetic field. Al-
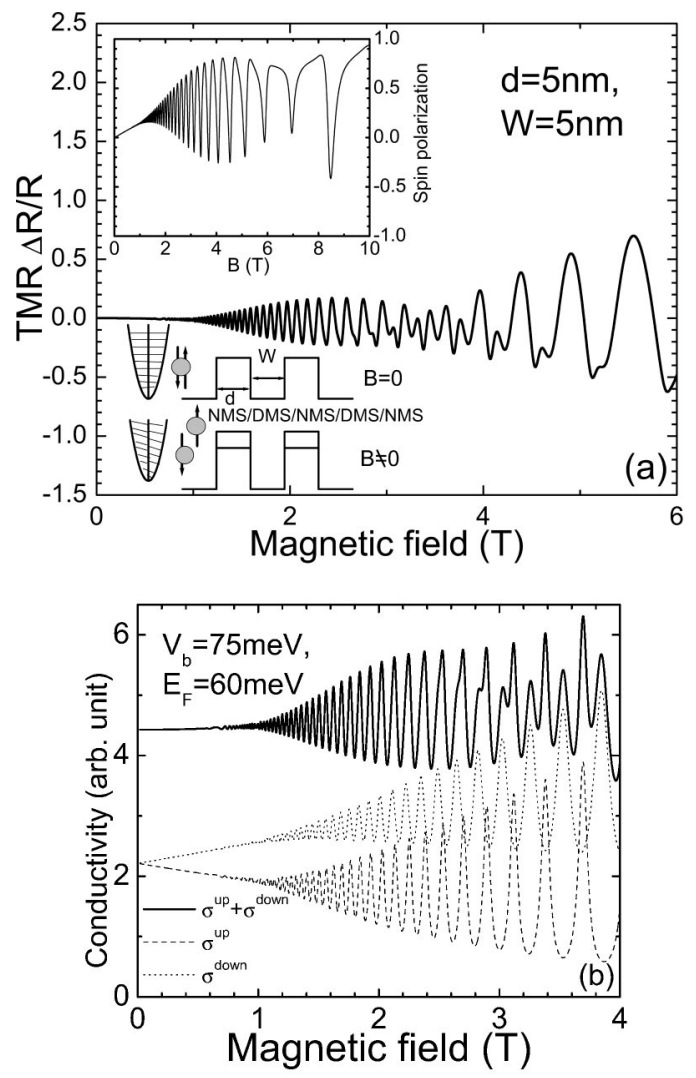

FIG. 4. The same as Figs. 3(a) and 3(b) but now for the DMS double barrier structure with NMS contacts. The parameters are

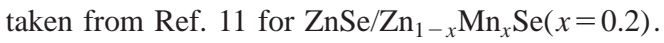


though the NMS and DMS materials are different from those of Ref. 13, the TMR still exhibits similar behavior with Fig. 2(a) of Ref. 13.

In Fig. 3(a), we find that the tunneling magnetoresistance (TMR) $\Delta R / R$ of a NMS double barrier (DB) structure with DMS contacts oscillates as a function of the magnetic field. A similar behavior is also observed in the spin polarization [see the inset of Fig. 3(a)]. A peculiar beat pattern is directly related to the superposition of the $s-d$ spin-split transmission channels which were found by Egues et al. ${ }^{13}$ Here we discuss this behavior in more detail. The oscillating behavior arises from the interplay between the Fermi surface and the position of the Landau levels of the two spin states. When the magnetic field increases, the Landau levels are swept across the Fermi surface one by one resulting in oscillations in the magnetoresistance whose origin is similar to those of Shubnikov de Haas ( $\mathrm{SdH}$ ) oscillations. In contrast to the usual $\mathrm{SdH}$ oscillations the $s-d$ exchange interaction leads to a giant Zeeman splitting at low temperature, and this splitting increases with increasing magnetic field which saturates for strong magnetic field which leads to an usual pattern of oscillations. The beating is a result of the fact that the total current is composed of spin-up and spin-down components which are split by the $s-d$ exchange interaction. The interplay between the spin-up and spin-down channels results in the beat pattern in the magnetoresistance. This is clearly demonstrated in Fig. 3(b) where we show the total conductivity (the thick curve) and the spin-up (dotted) and spindown (dashed) components. The phase difference of the oscillating conductivities of the spin-up and spin-down channels varies with increasing magnetic field, therefore the reduction and enhancement of the oscillating conductivity of the spin-up and spin-down channels lead to the beat pattern of the total TMR through the NMS double barriers with DMS contacts.

Figures 4(a) and 4(b) show the TMR $\Delta R / R$ as a function of magnetic field for DMS double barriers with NMS con- tacts which have not been studied in Ref. 13. The inset of Fig. 4(a) shows the spin polarization versus the magnetic field. Similar oscillating and beat behaviors as in Fig. 3(a) can be found in this figure. Comparing these results with Figs. 3(a) and 3(b), the beat patterns in the TMR and the SP are weakened since the difference between the spin-up and the spin-down electron conductivities varies slowly with increasing magnetic field for the DMS double barriers with NMS contacts.

In conclusion, we studied theoretically the $s$ - $d$ exchange interaction in DMS single and double barrier structures. Our theoretical results demonstrate that the oscillating TMR in DMS double barrier structures can be controlled by an external magnetic field which was first observed by Egues et al. ${ }^{12,13}$ The beat pattern in TMR arises from the interference between the spin-up and spin-down channels. The spin polarization also exhibits an oscillating behavior when the thickness of the DMS layer changes. Notice that the NMS/ DMS structures used in our calculation are already realized experimentally in recent magneto-optical studies, but at present

no transport measurements on such structures are available. Our results clearly illustrate that the spin polarization of the tunneling current can be tuned in magnitude and sign by changing the external magnetic field and/or the width of the tunneling barrier. Such systems are extremely attractive from the point of view of both basic research and technological applications, such as, e.g., in spin switches and spin transistors.

This work was financially supported by CAS, the Chinese Science Foundation, the Flemish Science Foundation (FWO-Vl), the Belgian Inter-University Attraction Poles program (IUAP), the Concerted Action program (GOA), the Inter-University Microelectronics Center (IMEC, vzw), and the Flemish-Chinese bilateral science and technological cooperation.
*Electronic address: kchang@red.semi.ac.cn

†Electronic address: peeters@uia.ua.ac.be

${ }^{1}$ S. Datta and B. Das, Appl. Phys. Lett. 56, 665 (1990).

${ }^{2}$ G.A. Prinz, Phys. Today 48, 58 (1995); G.A. Prinz, Science 282, 1660 (1998).

${ }^{3}$ B.E. Kane, Nature (London) 393, 133 (1998).

${ }^{4}$ D. Loss and D.P. DiVincenco, Phys. Rev. A 57, 120 (1998).

${ }^{5}$ M. Oestreich, J. Hubner, D. Hagele, P.J. Kar, W. Heimbrodt, W.W. Ruhle, D.E. Ashenford, and B. Lunn, Appl. Phys. Lett. 74, 1251 (1999).

${ }^{6}$ W.Y. Lee, S. Gardelis, B.C. Choi, Y.B. Xu, C.G. Smith, C.H.W. Barnes, D.A. Ritchie, E.H. Linfield, and J.A.C. Bland, J. Appl. Phys. 85, 6682 (1999).

${ }^{7}$ P.R. Hammar, B.R. Bennet, M.J. Yang, and M. Johnson, Phys. Rev. Lett. 83, 203 (1999).

${ }^{8}$ G. Schmidt, D. Ferrand, L.W. Molenkamp, A.T. Filip, and B.J. van Wees, Phys. Rev. B 62, R4790 (2000).

${ }^{9}$ J. Kossut, Phys. Status Solidi B 72, 359 (1975).

${ }^{10}$ M. von Ortenberg, Phys. Rev. Lett. 49, 1041 (1982).

${ }^{11}$ N. Dai, L.R. Ram-Mohan, H. Luo, G.L. Yang, F.C. Zhang, M.
Dobrowolska, and J.K. Furdyna, Phys. Rev. B 50, 18153 (1994).

12 J.C. Egues, Phys. Rev. Lett. 80, 4578 (1998).

${ }^{13}$ J.C. Egues, C. Gould, G. Richter, and L.W. Molenkamp, cond-mat/0103442.

${ }^{14}$ Y. Guo, H. Wang, B. Gu, and Y. Kawazoe, J. Appl. Phys. 88, 6614 (2000).

${ }^{15}$ Kai Chang and F. M. Peeters, Solid State Commun. 120, 181 (2001).

${ }^{16}$ R. Fiederling, M. Keim, G. Reuscher, W. Ossau, G. Schmidt, A. Waag, and L.W. Molenkamp, Nature (London) 402, 787 (1999).

${ }^{17}$ Y. Ohno, D.K. Young, B. Benschoten, F. Matsukura, H. Ohno, and D.D. Awschalom, Nature (London) 402, 790 (1999).

${ }^{18}$ J.M. Kikkawa, I.P. Smorchkova, N. Samarth, and D.D. Awshalom, Science 277, 1284 (1997); J.M. Kikkawa and D.D. Awshalom, Nature (London) 397, 139 (1999); J.M. Kikkawa and D.D. Awschalom, Phys. Rev. Lett. 80, 4313 (1998).

${ }^{19}$ G. Bastard and R. Ferreira, Surf. Sci. 267, 335 (1992).

${ }^{20}$ M. Herbich, A. Twardowski, D. Scalbert, and A. Petrou, Phys. Rev. B 58, 7024 (1998). 
${ }^{21}$ J.A. Gaj, R.R. Gatazka, and M. Nawrocki, Solid State Commun. 25, 193 (1978); W. Ossau, U. Zehnder, B. Kuhn-Heinrich, A. Waag, Th. Litz, and G. Landwehr, Superlattices Microstruct. 16, 5 (1994); B. Kuhn-Heinrich, W. Ossau, H. Heinke, F. Fischer, T. Litz, A. Waag, and G. Landwehr, Appl. Phys. Lett. 63, 2932
(1993); W.J. Ossau and B. Kuhn-Heinrich, Physica B 184, 422 (1993); W. Grieshaber, A. Haury, J. Cibert, Y. Merle d̆Aubignè, A. Wasiela, and J.A. Gaj, Phys. Rev. B 53, 4891 (1996).

${ }^{22}$ G.E. Blonder, M. Tinkham, and T.M. Klapwijk, Phys. Rev. B 25, 4515 (1982). 